Case Report

\title{
Double-Hit Primary Plasma Cell Leukemia with IGH/MYC and IGH/CCND1 Translocations
}

\author{
Masato Yasumi (D), ${ }^{1}$ Takaya Endo, ${ }^{1}$ Hiroshi Sata, ${ }^{2}$ and Takahiro Karasuno ${ }^{1}$ \\ ${ }^{1}$ Department of Hematology, Rinku General Medical Center, Izumisano, Japan \\ ${ }^{2}$ Division of Hematology, Department of Internal Medicine, Daini Osaka Police Hospital, Osaka, Japan \\ Correspondence should be addressed to Masato Yasumi; m-yasumi@rgmc.izumisano.osaka.jp
}

Received 10 June 2020; Revised 27 November 2020; Accepted 4 December 2020; Published 18 December 2020

Academic Editor: Sergio Storti

Copyright (C) 2020 Masato Yasumi et al. This is an open access article distributed under the Creative Commons Attribution License, which permits unrestricted use, distribution, and reproduction in any medium, provided the original work is properly cited.

\begin{abstract}
Primary plasma cell leukemia (pPCL) is an aggressive variant of multiple myeloma (MM). Immunoglobulin heavy chain (IgH) translocations are found in a majority of pPCL cases, supporting a central relation to pathogenesis of pPCL. However, two independent $I g H$ translocations are barely detected at the onset of PPCL, and their significance is yet to be elucidated. Here, we report a case of an aggressive pPCL with simultaneous IGH/MYC and IGH/CCND1 translocations. A 73-year-old man was referred to our hospital with back pain and diagnosed as having pPCL with more than $50 \%$ circulating plasma cells. Cytogenetic analysis revealed 47, Y, t (X; 8;14) (q24; q24; q32), t $(11 ; 14)(\mathrm{q} 13 ; \mathrm{q} 32)$, and +18. IGH/MYC and IGH/CCND1 translocations were confirmed by fluorescence in situ hybridization analysis. Bortezomib and dexamethasone treatment achieved rapid elimination of peripheral malignant plasma cells, and the patient maintained a partial response for 18 months. After biological relapse, he received salvage therapy with ixazomib, lenalidomide, and dexamethasone, followed by pomalidomide and dexamethasone, and exhibited stable disease for an additional 14 months. Although IGH/MYC translocation in association with dysregulation of antiapoptotic pathway leads to worse prognosis in lymphomas, the novel agent-based regimen showed good efficacy, suggesting that IGH/MYC plays a different role in the pathogenesis of MM. IGH/CCND1 and IGH/MYC translocations may have contributed to abrupt onset of PPCL in this case.
\end{abstract}

\section{Introduction}

Plasma cell leukemia (PCL) is a rare and aggressive form of multiple myeloma (MM), and the incidence of PCL was reported to be less than $1 \%$ of MM cases [1]. PCL is classified into two clinical types: primary PCL (pPCL) and secondary PCL [2]. In the former type, presentation is de novo and the prognosis is poor, with median overall survival (OS) of 4 months due to being refractory to various chemotherapies [1].

Recurrent immunoglobulin heavy chain $(\operatorname{IgH})$ gene translocations involving CCND1, FGFR3 and MMEST, $C C N D 3, M A F$, and MAFB are important oncogenic pathways observed in about $40 \%$ of MM and in $87 \%$ of pPCL cases [3]. Among them, CCND1 is the most prevalent partner of $I g H$ translocation, detected in $20-71 \%$ of pPCL cases [4]. Because most of these primary $I g H$ translocations are speculated to be mediated by errors in $I g H$ class switch recombination during $\mathrm{B}$ cell maturation, they are considered to be an early oncogenic event [3]. On the other hand, $\operatorname{IgH}$ translocations that target $M Y C$ are thought to be a very late and progressive event in the course of $\mathrm{MM}$ and rarely identified at the onset of MM, especially in PPCL [5].

Here, we report a case of an aggressive pPCL with simultaneous IGH/MYC and IGH/CCND1 translocations.

\section{Case Report}

A 73-year-old man was referred to our hospital with a history of back pain for one month. Laboratory findings were as follows: a white blood cell count of $8.9 \times 109 / \mathrm{L}$ with $55.0 \%$ of atypical plasma cells, hemoglobin level of $8.1 \mathrm{~g} / \mathrm{dL}$, platelet count of $55 \times 109 / \mathrm{L}$, lactate dehydrogenase level of $326 \mathrm{IU} / \mathrm{L}$, creatinine level of $1.03 \mathrm{mg} / \mathrm{dL}$, and blood urea 
nitrogen of $30.6 \mathrm{mg} / \mathrm{dL}$. IgG was elevated at $4847 \mathrm{mg} / \mathrm{dL}$ with low levels of IgA and IgM $(31 \mathrm{mg} / \mathrm{dL}$ and $9 \mathrm{mg} / \mathrm{dL}$, respectively), and free light chain (FLC) analysis demonstrated a slight increase in free $\lambda$ chain: $\kappa$ FLC of $3.1 \mathrm{mg} / \mathrm{dL}, \lambda$ FLC of $50.8 \mathrm{mg} / \mathrm{dL}$, and $\kappa / \lambda$ ratio of 0.06 .

Immunoelectrophoresis of serum identified IgG- $\lambda$ monoclonal protein. Bone marrow examination revealed an infiltration of atypical plasma cells, comprising 55\% of overall cellularity. Cytogenetic analysis revealed 47, Y, t (X; 8 ; 14) (q24; q24; q32), t $(11 ; 14)$ (q13; q32), and +18 in 2 of the 20 cells studied. On immunohistochemistry, atypical plasma cells were strongly positive for cyclin D1 and partially weakly positive for MYC (Figure 1). We confirmed $I G H / M Y C$ and IGH/CCND1 translocations by fluorescence in situ hybridization (FISH) analysis at the first relapse (Figure 2). Thus, he was diagnosed as having IgG- $\lambda$ type primary plasma cell leukemia with IGH/MYC and IGH/ CCND1 translocations.

Initially, the patient was treated by Bd therapy $(1.3 \mathrm{mg} /$ $\mathrm{m} 2$ of bortezomib administered subcutaneously on days 1,8 , and 15 every 28 days, with $20 \mathrm{mg}$ dexamethasone administered orally on days 1-2, 8-9, and 15-16). Malignant plasma cells rapidly disappeared from peripheral blood after one cycle of Bd therapy. He achieved a partial response after three cycles of Bd therapy. His IgG level was stable at around $1200 \mathrm{mg} / \mathrm{dL}$ for 18 months; however, it increased to $3083 \mathrm{mg} / \mathrm{dL}$ after 15 cycles of Bd therapy. As second-line therapy, IRd therapy ( $4 \mathrm{mg}$ of ixazomib given orally on days 1,8 , and 15 every 28 days in addition to reduced doses of lenalidomide (10 $\mathrm{mg}$ daily on days 1-21) and $20 \mathrm{mg}$ of dexamethasone weekly) was administered. IRd therapy was effective, and his IgG level decreased to $1380 \mathrm{mg} / \mathrm{dL}$ after five cycles. He achieved a partial response again. Unfortunately, he fractured the right femoral neck after 12 cycles of IRd therapy. He also developed severe pneumonia; therefore, we discontinued IRd therapy. Thereafter, bone marrow examination was performed, which revealed increased atypical plasma cells, and additional aberrations, including der (11)t $(11 ; 14)$ and der $(14) t(8 ; 14)$, were found on cytogenetic analysis (Figure 2(b) and Table 1). Due to his poor performance status and cytopenia, reduced-dose Pd therapy ( $1 \mathrm{mg}$ of pomalidomide administered daily on days 1-14 every 28 days with $8 \mathrm{mg}$ of dexamethasone weekly) was started. His IgG level had been stable at around $2000 \mathrm{mg} / \mathrm{dL}$ for 5 months. However, he developed recurrent infections and became bedridden, and then, he received palliative care and died 36 months after diagnosis.

\section{Discussion}

We report a case of pPCL harboring IGH/MYC and IGH/ CCND1 translocations at onset that showed a good response to novel agent-based treatment.

There are two main types of primary cytogenetic abnormalities in MM: hyperdiploidy and primary $\mathrm{IgH}$ translocations. In pPCL, IgH translocations were observed in $87 \%$, and CCND1 was the most common translocation partner observed in 20-71\% [4], supporting a central role of $I G H / C C N D 1$ translocation in the pathogenesis of pPCL.
Recently, as high $B C L 2 / B C L-X_{L}$ and $M C L-1$ expression ratios were reported in $\mathrm{t}(11 ; 14) \mathrm{MM}$, IGH/CCND1 translocation may contribute to plasma cell survival by altering the antiapoptotic pathway [7].

$M Y C$ is dysregulated or overexpressed in most human cancers and contributes to cell activation, cell proliferation, and apoptosis [8]. In MM, upregulation of $M Y C$ is involved in the progression from MGUS to MM [9] and also associated with leukemic and extramedullary presentation [10]. MYC is dysregulated by genetic rearrangements, such as translocations and amplifications, and is also modulated by deregulation of upstream pathways such as IRF4, DIS3/ LIN28B/let-7, or MAPK [11].

Ig translocation with $M Y C$ is speculated to be a late and progressive event [12] and has been reported in $2.4 \%$ of newly diagnosed MM cases and rarely reported at the onset of pPCL [5]. To our knowledge, double translocations of $\operatorname{IgH}$ with $M Y C$ and CCND1 were reported in only six cases of newly diagnosed MM, and this is the second case in PPCL [5, 13-16]. Nakayama et al. reported a 76-year-old patient with double-hit nonsecretory pPCL who had advanced disease [16], and Ji et al. described a 51-year-old patient with doublehit IgD myeloma who exhibited an aggressive clinical course and became refractory to conventional chemotherapy 18 months after the diagnosis [13]. However, there was no description of the detailed clinical course in other previously reported myeloma cases. Therefore, this is the first report of a clinical response to novel agent-based treatment.

Double-hit lymphoma with rearrangements of $M Y C$ and BCL2 is recognized as a distinct entity under the WHO 2016 and known to have very poor prognosis [17]. Concomitant dysregulation of antiapoptotic molecule BCL2 may counteract the proapoptotic function of $M Y C$, resulting in rapid cell growth and resistance to therapy [18]. However, the prognosis of a double-hit case with rearrangements of $M Y C$ and CCND1 in pPCL is not known, especially when treated with novel agent-based regimens. Despite leukemic presentation, frailty, as well as MYC and CCND1 rearrangements, doublet and triplet regimens of novel agents had good efficacy in the patient and responded to treatment for more than 30 months after diagnosis. Recently, it was reported that IGH/MYC translocations in MM have extragenic $I G H$ breakpoints, which are distinct from other B cell malignancies that usually have breakpoints in $I G H$ switch regions [6]. These genetic alterations and cell differentiation states may contribute to affect the relatively good treatment response in IGH/MYC-possessing MM.

Interestingly, a previous study observed that cyclin $D$ genes (cyclin D1, D2, and D3) were ectopically expressed in almost all MM cases from an early stage, implying a unifying and initial oncogenic event in MM [19]. In this case, juxtaposition of CCND1 and MYC to the $\mathrm{IgH}$ gene enhancer may lead to simultaneous activation of initiating and progressive oncogenic pathways, resulting in abrupt onset of aggressive pPCL.

We also observed the accumulation of IGH/MYC and $I G H / C C N D 1$ translocations over time during treatment, which suggested that $M Y C$ and CCND1 signaling play a role in disease progression. There were discrepancies between 


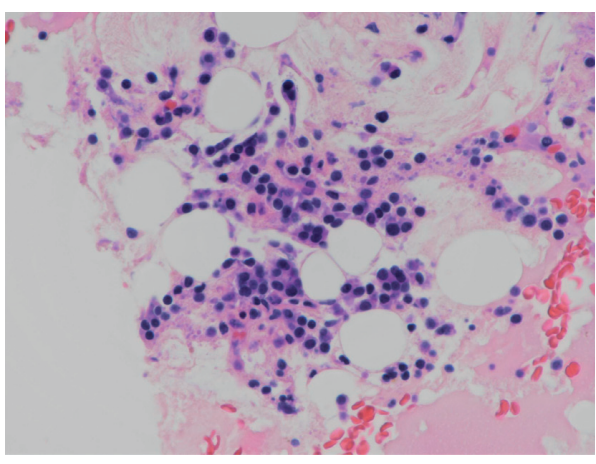

(a)

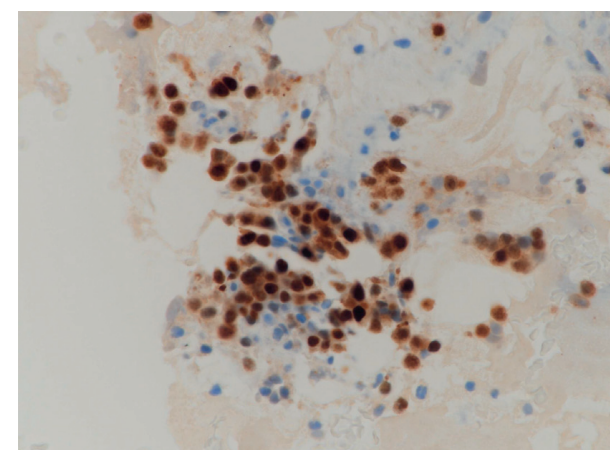

(b)

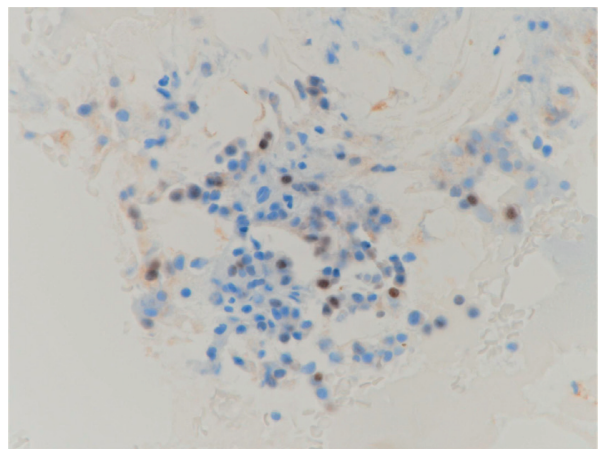

(c)

Figure 1: Pathological and immunohistochemical findings of bone marrow clot samples. (a) Hematoxylin and eosin staining showing infiltration of small-sized atypical plasma cells with eccentric nuclei. (b) Plasma cells were strongly positive for cyclin D1. (c) Plasma cells exhibited partial and weak MYC expression. Magnification: 400x.

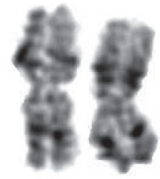

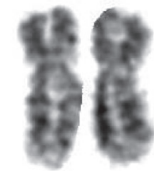

2

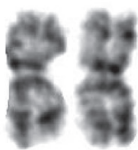

3

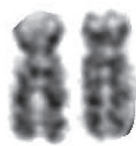

4

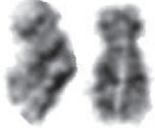

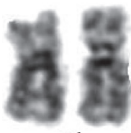

6
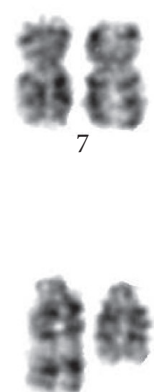

14

$\uparrow \uparrow$
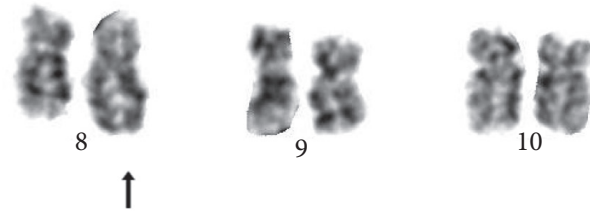

10

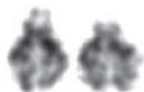

15

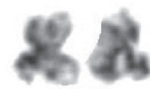

16

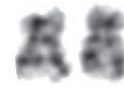

17
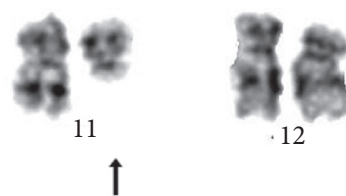

12

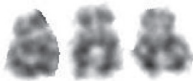

18

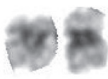

19
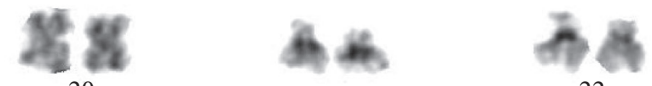

21

22

(a)

Figure 2: Continued. 


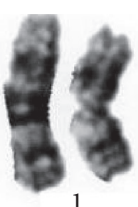

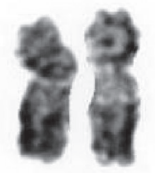

2

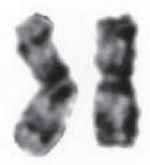

3

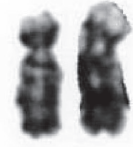

4

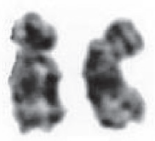

5

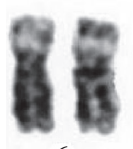

6

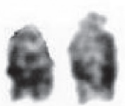

13

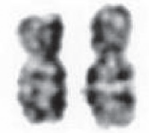

7

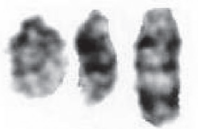

14

$\uparrow \uparrow \uparrow$

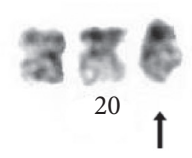

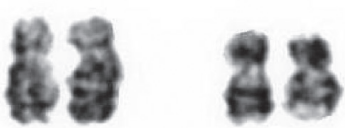

8

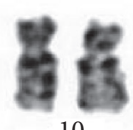

10

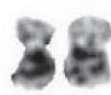

16

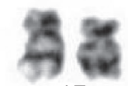

17

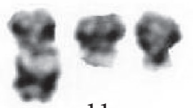

11

$\uparrow \uparrow$
12
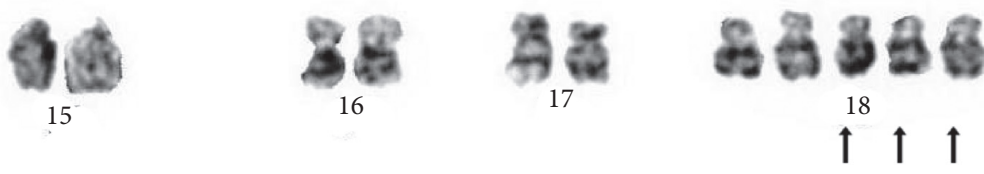

18
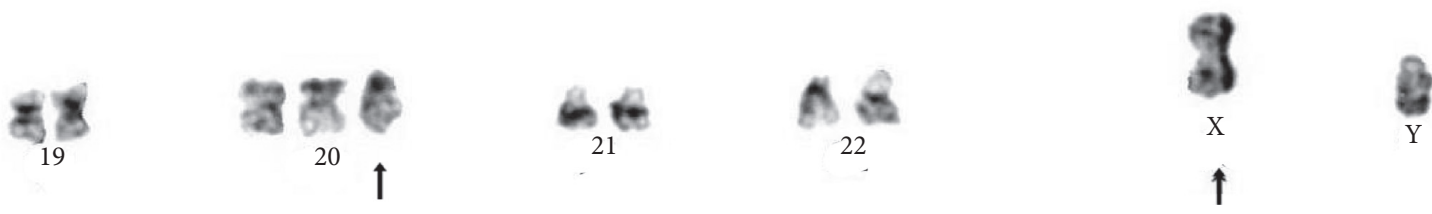

(b)

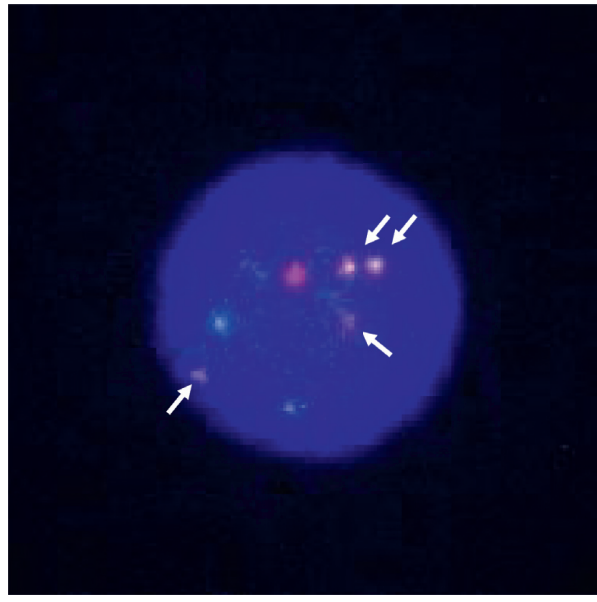

(c)

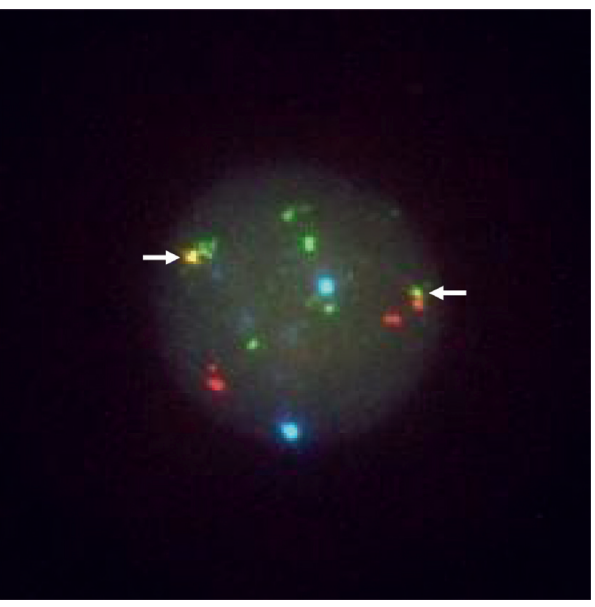

(d)

FIGURE 2: Karyotype of metaphase chromosomes and interphase fluorescence in situ hybridization (FISH) analysis obtained from the bone marrow. (a) The following complex karyotypes were observed at diagnosis: 47, Y, t (X; 8; 14) (q24; q24; q32), t (11; 14) (q13; q32), and +18. The arrows indicate the abnormal chromosomes. (b) Additional karyotype aberrations, including der $(11) \mathrm{t}(11 ; 14)$ and der $(14) \mathrm{t}(8 ; 14)$, were observed after IRd therapy. (c) FISH analysis was performed using a vysis LSI IGH/CCND1 dual color, dual fusion translocation probe. The orange signal represents CCND1, and the green signal represents IGH. Four fusion signals were observed. (d) FISH analysis was performed using a vysis LSI IGH/MYC/CEP 8 tri-color dual fusion probe. The orange signal represents MYC, the green signal represents IGH, and the aqua signal represents alpha satellite sequences on 8q11.1. Two fusion signals were visible. 
TABLE 1: Development of cytogenetic aberrations over time during treatment.

\begin{tabular}{|c|c|}
\hline Period & Karyotype Result \\
\hline At onset & $47, \mathrm{Y}, \mathrm{t}(\mathrm{X} ; 8 ; 14)(\mathrm{q} 24 ; \mathrm{q} 24 ; \mathrm{q} 32), \mathrm{t}(11 ; 14)(\mathrm{q} 13 ; \mathrm{q} 32),+18[2] / 46, \mathrm{XY}[6]$ \\
\hline $\begin{array}{l}\text { At first relapse (after Bd } \\
\text { therapy) }\end{array}$ & $51, \mathrm{Y}, \mathrm{t}(\mathrm{X} ; 8 ; 14)(\mathrm{q} 24 ; \mathrm{q} 24 ; \mathrm{q} 32),-5, \mathrm{t}(11 ; 14)(\mathrm{q} 13 ; \mathrm{q} 32),+12,-17,+18+20,+4 \operatorname{mar}[1] / 46, \mathrm{XY}[5]$ \\
\hline After IRd therapy & 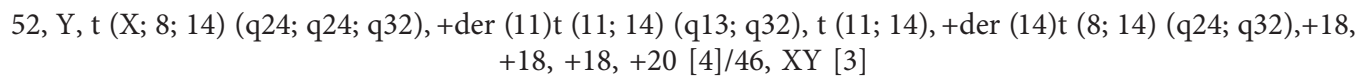 \\
\hline
\end{tabular}

cytogenetic analysis and FISH analysis in the time period and number of additional translocations. These may reflect the high sensitivity of FISH analysis in detecting chromosomal translocations [20].

Here, we described a case of double-hit primary plasma cell leukemia with $I G H / M Y C$ and $I G H / C C N D 1$ successfully treated with novel agent-based regimens.

It is important to evaluate the MYC and CCND1 signaling in the pathogenesis of pPCL for further understanding and identifying new therapeutic targets of pPCL.

\section{Conflicts of Interest}

The authors declare that they have no conflicts of interest.

\section{References}

[1] W. I. Gonsalves, S. V. Rajkumar, R. S. Go et al., "Trends in survival of patients with primary plasma cell leukemia: a population-based analysis," Blood, vol. 124, no. 6, pp. 907-912, 2014.

[2] International Myeloma Working Group, "Criteria for the classification of monoclonal gammopathies, multiple myeloma and related disorders: a report of the international myeloma working group," British Journal Haematology, vol. 121, pp. 749-757, 2003.

[3] M. Chesi and P. L. Bergsagel, "Molecular pathogenesis of multiple myeloma: basic and clinical updates," International Journal of Hematology, vol. 97, no. 3, pp. 313-323, 2013.

[4] C. F. De Larrea, R. A. Kyle, B. G. M. Durie et al., "Plasma cell leukemia: consensus statement on diagnostic requirements, response criteria, and treatment recommendations by the international myeloma working group (IMWG)," Leukemia, vol. 27, pp. 780-791, 2013.

[5] H. Avet-Loiseau, F. Gerson, F. Magrangeas, S. Minvielle, J. L. Harousseau, and R. Bataille, "Rearrangements of the c-myc oncogene are present in $15 \%$ of primary human multiple myeloma tumors," Blood, vol. 98, no. 10, pp. 30823086, 2001.

[6] B. G. Barwick, P. Neri, N. J. Bahlis et al., "Multiple myeloma immunoglobulin lambda translocations portend poor prognosis," Nature Communications, vol. 10, no. 1, p. 1911, 2019.

[7] S. Kumar, J. L. Kaufman, C. Gasparetto, J. Mikhael et al., "Efficacy of venetoclax as targeted therapy for relapsed/refractory t $(11 ; 14)$ multiple myeloma," Blood, vol. 130, no. 22, pp. 2401-2409, 2017.

[8] C. V. Dang, "Myc on the path to cancer," Cell, vol. 149, no. 1, pp. 22-35, 2012.

[9] W.-J. Chng, G. F. Huang, T. H. Chung et al., "Clinical and biological implications of MYC activation: a common difference between MGUS and newly diagnosed multiple myeloma," Leukemia, vol. 25, no. 6, pp. 1026-1035, 2011.
[10] I. C. Glitza, G. Lu, R. Shah et al., "Chromosome 8q24.1/ c-MYCabnormality: a marker for high-risk myeloma," Leukemia \& Lymphoma, vol. 56, no. 3, pp. 602-607, 2014.

[11] K. K. Jovanovic, C. Roche-Lestienne, I. M. Ghobrial, T. Facon, B. Quesnel, and S. Manier, "Targeting MYC in multiple myeloma," Leukemia, vol. 32, pp. 1295-1306, 2018.

[12] A. Dib, A. Gabrea, O. K. Glebov, P. L. Bergsagel, and W. M. Kuehl, "Characterization of MYC translocations in multiple myeloma cell lines," JNCI Monographs, vol. 2008, no. 39, pp. 25-31, 2008.

[13] M. Ji, S. Jang, J.-H. Lee, and E.-J. Seo, "Double-hit myeloma with IGH/MYC and IGH/CCND1 translocations," Annals of Hematology, vol. 92, no. 8, pp. 1129-1131, 2013.

[14] N. V. Smadja, C. Bastard, C. Brigaudeau, D. Leroux, and C. Fruchart, "Hypodiploidy is a major prognostic factor in multiple myeloma," Blood, vol. 98, no. 7, pp. 2229-2238, 2001.

[15] J. L. Lai, L. Michaux, N. Dastugue et al., "Cytogenetics in multiple myeloma: a multicentric study of 24 patients with $\mathrm{t}$ $(11 ; 14)$ (q13; q32) or its variant," Cancer Genet Cytogenet, vol. 104, pp. 133-138, 1998.

[16] S. Nakayama, T. Oda, S. Minabe et al., "Primary nonsecretory plasma cell leukemia with multiple chromosomal abnormalities: a case report," Archives of Iranian Medicine, vol. 21, no. 1, pp. 41-43, 2018.

[17] S. H. Swerdlow, E. Campo, S. A. Pileri et al., "The 2016 revision of the world health organization classification of lymphoid neoplasms," Blood, vol. 127, no. 20, pp. 2375-2390, 2016.

[18] G. Ott, "Impact of MYC on malignant behavior," Hematology, vol. 2014, no. 1, pp. 100-106, 2014.

[19] P. L. Bergsagel, W. M. Kuehl, F. Zhan, J. Sawyer, B. Barlogie, and J. Shaughnessy, "Cyclin D dysregulation: an early and unifying pathogenic event in multiple myeloma," Blood, vol. 106, no. 1, pp. 296-303, 2005.

[20] K. Nishida, A. Tamura, N. Nakazawa et al., "The Ig heavy chain gene is frequently involved in chromosomal translocations in multiple myeloma and plasma cell leukemia as detected by in situ hybridization," Blood, vol. 90, no. 2, pp. 526-534, 1997. 